\title{
Geometrical aspects of subspace codes
}

\author{
Antonio Cossidente and Francesco Pavese and Leo Storme
}

\begin{abstract}
Subspace codes are codes whose codewords are equal to subspaces of a finite vector space $V(n, q)$. Since the geometry of the subspaces of a finite vector space $V(n, q)$ is equivalent to the geometry of the subspaces of a projective space $\operatorname{PG}(n-1, q)$, problems on subspace codes can be investigated by using geometrical arguments. Here, we illustrate this approach by showing some recent results on subspace codes, obtained via geometrical arguments. We discuss upper bounds on the sizes of subspace codes, by showing the link between the Johnson bound and the size of partial spreads in finite projective spaces. We present geometrical constructions of subspace codes, and we also focus on subspace codes constructed from Maximum Rank Distance (MRD) codes. Here, we also present geometrical links of MRD codes to exterior sets of Segre varieties. Our aim is to motivate researchers on subspace codes to also consider geometrical arguments when investigating problems on subspace codes.
\end{abstract}

\section{Introduction}

The finite projective space $\mathrm{PG}(n-1, q)$ of dimension $n-1$ over the finite field $\mathbb{F}_{q}$ of order $q$ is constructed from the vector space $V(n, q)$ of dimension $n$ over the finite field $\mathbb{F}_{q}$ of order $q$ in the following way: an $i$-dimensional projective subspace

Antonio Cossidente

Dipartimento di Matematica e Informatica, Università della Basilicata, Contrada Macchia Romana, 85100 Potenza, Italy, e-mail: antonio.cossidente@unibas.it

Francesco Pavese

Dipartimento di Meccanica, Matematica e Management, Politecnico di Bari, Via Orabona 4, 70126, Bari, Italy, e-mail: francesco.pavese @ poliba.it

Leo Storme

Ghent University, Department of Mathematics, Krijgslaan 281, 9000 Ghent, Belgium, e-mail: leo.storme@ugent.be 
of $\mathrm{PG}(n-1, q)$ is defined by an $(i+1)$-dimensional subspace of the vector space $V(n, q)$.

R. Kötter and F. Kschischang [31] proved that a very good way of transmission in networks is obtained if subspace codes are used. Here, the codewords are subspaces of the $n$-dimensional vector space $V(n, q)$ over the finite field $\mathbb{F}_{q}$ of order $q$. To transmit a codeword, i.e. a $k$-dimensional vector space, through the network, it is sufficient to transmit a basis of this $k$-dimensional vector space. But a $k$-dimensional subspace has different bases. Kötter and Kschischang proved that the transmission can be optimalized if the nodes in the network transmit linear combinations of the incoming basis vectors of the $k$-dimensional subspace which represents the codeword.

The geometry of the subspaces of a vector space over a finite field can be investigated in the setting of finite projective spaces, also called Galois geometries. The three standard references $[24,25,26]$ to Galois geometries contain a wealth of information on Galois geometries. In Galois geometries, geometrical methods, and also other methods, such as polynomial methods and links with other domains, can be used to investigate a great variety of problems on substructures of these finite projective spaces. Since a subspace code precisely is a substructure of a finite projective space, this setting has, and still is able, to provide many new interesting results on subspace codes.

This article wishes to highlight this fact. We present some constructions of, and results on, subspace codes, which, presently, are among the best results, and which match results that are found via other techniques. We have concentrated on the constructions and results which can illustrate in an optimal way the ideas which have been used, and which show that Galois geometries and geometrical techniques are of great value for the study of subspace codes. We hope via these examples to motivate many researchers to also investigate problems on subspace codes via geometrical techniques, and to help showing that the study of Galois geometries is not only of purely theoretical importance, but also of practical importance.

We first present the basic ideas on Galois geometries to fix notations and properties. We then present these particular examples of results on subspace codes, obtained via geometrical arguments.

\section{Preliminaries}

Let $\mathbb{F}_{q}$ be the finite field of order $q$ and let $V(n, q)$ be the vector space of dimension $n$ over $\mathbb{F}_{q}$. The 1-dimensional subspaces of $V(n, q)$ are called vector lines, the 2 -dimensional subspaces are called vector planes. The number of $k$-dimensional subspaces of $V(n, q)$ is the $q$-binomial coefficient

$$
\left[\begin{array}{l}
n \\
k
\end{array}\right]_{q}=\frac{\left(q^{n}-1\right)\left(q^{n-1}-1\right) \cdots\left(q^{n-k+1}-1\right)}{\left(q^{k}-1\right)\left(q^{k-1}-1\right) \cdots(q-1)} .
$$


The number of $k$-dimensional subspaces containing a fixed $t$-dimensional subspace, $t \leq k$, is

$$
\left[\begin{array}{l}
n-t \\
k-t
\end{array}\right]_{q}
$$

The subspace distance between subspaces $U$ and $W$ of $V(n, q)$ is defined by

$$
d(U, W)=\operatorname{dim}(U+W)-\operatorname{dim}(U \cap W) .
$$

A subspace code $\mathscr{C}$ is a non-empty collection of subspaces of $V(n, q)$. A codeword is an element of $\mathscr{C}$. The minimum distance of $\mathscr{C}$ is defined by

$$
d(\mathscr{C}):=\min \{d(U, W) \mid U, W \in \mathscr{C}, U \neq W\} .
$$

A code $\mathscr{C}$ is called an $(n, M, d)$-subspace code if $\mathscr{C}$ consists of subspaces of $V(n, q)$, is of cardinality $M$, and has minimum distance $d$. The maximal number of codewords in an $(n, M, d)$-code, defined over $\mathbb{F}_{q}$, is denoted by $\mathscr{A}_{q}(n, d)$. A constant dimension subspace code is a code with all codewords of the same dimension. If all codewords of an $(n, M, d)$-code $\mathscr{C}$ are $k$-dimensional subspaces of $V(n, q)$, then we say that $\mathscr{C}$ is an $(n, M, d, k)$-subspace code. The maximum number of codewords in an $(n, M, d, k)$-code, defined over $\mathbb{F}_{q}$, is denoted by $\mathscr{A}_{q}(n, d, k)$.

\subsection{Codes in projective spaces}

We will investigate subspace codes in the projective setting.

The projective space $\operatorname{PG}(n, q)$ of projective dimension $n$ over $\mathbb{F}_{q}$ is the lattice of subspaces of $V(n+1, q)$ with respect to set inclusion. In this article, a $k$-space, $0 \leq k \leq n$, is a subspace $\mathrm{PG}(k, q)$ of $\mathrm{PG}(n, q)$ of projective dimension $k$. This means that a projective $k$-space is defined by a $(k+1)$-dimensional subspace of $V(n+1, q)$, but we interpret the vector lines contained in this $(k+1)$-dimensional subspace as 0 dimensional objects, called projective points. The dimension $k$ of a projective space $\mathrm{PG}(k, q)$ is called the projective dimension of this subspace $\mathrm{PG}(k, q)$.

The number of projective points of a $k$-space is $\left[\begin{array}{c}k+1 \\ 1\end{array}\right]_{q}$. An $i$-dimensional projective space $\mathrm{PG}(i, q), i=1,2,3, n-1$, is called a projective line, a projective plane, a solid or a hyperplane of $\mathrm{PG}(n, q)$, respectively.

Alternatively, $\operatorname{PG}(n, q)$ can be defined as a point-line geometry [15]. Let $\Delta$ be a $k$-space of $\mathrm{PG}(n, q)$. If the subspaces of $\mathrm{PG}(n, q)$ of dimension $k+1$ and $k+2$, containing $\Delta$, are considered as new points and lines, with inclusion as incidence, then they correspond to a projective geometry $\mathrm{PG}(n-k-1, q)$, called the quotient geometry of $\Delta$. We shall denote the quotient geometry of $\Delta$ as $\mathrm{PG}(n-k-1, q)_{\Delta}$. An $m$-space of $\operatorname{PG}(n, q)$ that contains $\Delta$ has projective dimension $m-k-1$ in $\operatorname{PG}(n-$ $k-1, q)_{\Delta}$.

To illustrate the close links between subspace codes and finite projective spaces, we illustrate how the study of the Johnson bound immediately relates to the study 
of a geometrical object in finite projective spaces, called a partial spread, which is already investigated for many years in Galois geometries.

\section{Johnson bound and partial spreads in finite projective spaces}

\subsection{The Johnson bound and partial spreads}

Definition 1. A $(k-1)$-spread of $\operatorname{PG}(n-1, q)$ is a partitioning of the point set of $\mathrm{PG}(n-1, q)$ in $(k-1)$-spaces.

A partial $(k-1)$-spread $\mathscr{S}$ of $\mathrm{PG}(n-1, q)$ is a set of pairwise disjoint $(k-1)$ spaces of $\mathrm{PG}(n-1, q)$.

A $(k-1)$-spread of $\operatorname{PG}(n-1, q)$ exists if and only if $n \equiv 0(\bmod k)$. Then, the size of a $(k-1)$-spread is $\left[\begin{array}{l}n \\ 1\end{array}\right]_{q} /\left[\begin{array}{l}k \\ 1\end{array}\right]_{q}$.

Partial spreads are closely related to subspace codes. A $(k-1)$-spread of $\mathrm{PG}(n-$ $1, q)$ is a maximal $(n, M, 2 k, k)$-code of cardinality

$$
M=\mathscr{A}_{q}(n, 2 k, k)=\left[\begin{array}{l}
n \\
1
\end{array}\right]_{q} /\left[\begin{array}{l}
k \\
1
\end{array}\right]_{q}, \quad \text { when } n \equiv 0 \quad(\bmod k) .
$$

A partial $(k-1)$-spread of $\operatorname{PG}(n-1, q), n \equiv 0(\bmod k)$, of size $M$ is also an $(n, M, 2 k, k)$-code, not necessarily of the maximal size.

When $k$ does not divide $n$, the following lower bound on $\mathscr{A}_{q}(n, 2 k, k)$ is known.

Theorem 1. [6, 20] Let $n \equiv r(\bmod k), 0 \leq r \leq k-1$. Then for all $q$, we have

$$
\mathscr{A}_{q}(n, 2 k, k) \geq \frac{q^{n}-q^{k}\left(q^{r}-1\right)-1}{q^{k}-1} .
$$

The following upper bound on $\mathscr{A}_{q}(n, 2 k, k)$ is known.

Theorem 2. [5, 17] Let $\mathscr{S}$ be a partial $t$-spread in $\operatorname{PG}(d, q)$, where $d=k(t+1)-$ $1+r, 1 \leq r \leq t$.

Let $|\mathscr{S}|=q^{r} \cdot \frac{q^{k(t+1)}-1}{q^{t+1}-1}-s$. Then

- $s \geq q-1$.

- $s>\frac{q^{r}-1}{2}-\frac{q^{2 r-t-1}}{5}$.

Since Theorem 1 states that there exists an example with $s=q^{r}-1$, we know that

$$
\mathscr{A}_{q}(n, 2 k, k)=\frac{q^{n}-q^{k}\left(q^{r}-1\right)-1}{q^{k}-1},
$$

when $r=1$. 
For many years, it was conjectured that

$$
\mathscr{A}_{q}(n, 2 k, k)=\frac{q^{n}-q^{k}\left(q^{r}-1\right)-1}{q^{k}-1},
$$

so the lower bound of Theorem 1 is sharp.

However, El-Zanati et al [18] found a counter example: there is a partial 3spread in the finite vector space $V(8,2)$, which corresponds to a partial 2-spread in $\mathrm{PG}(7,2)$, of size 34 , but there exists no example of size 35 . So $\mathscr{A}_{2}(8,6,3)=34$.

But, very recently, a major breakthrough has been made by E. Năstase and P. Sissokho [37].

Theorem 3. [37] Let $r \equiv n(\bmod k)$. Then, if

$$
k>\frac{q^{r}-1}{q-1},
$$

the maximal size for a partial $(k-1)$-spread in $\mathrm{PG}(n-1, q)$ is equal to $\frac{q^{n}-q^{k}\left(q^{r}-1\right)-1}{q^{k}-1}$.

Consequently, if $k>\frac{q^{r}-1}{q-1}$, then

$$
\mathscr{A}_{q}(n, 2 k, k)=\frac{q^{n}-q^{k}\left(q^{r}-1\right)-1}{q^{k}-1} .
$$

Similar results for $q=2$ were recently also proven by S. Kurz [32].

We now make the link between the problem of the largest size of partial spreads in finite projective spaces to the Johnson bound on subspace codes.

In general, in the terminology of projective geometry, an $(n, M, d, k)$-code $\mathscr{C}$ can be viewed as a set of $(k-1)$-spaces of $\mathrm{PG}(n-1, q)$ of cardinality $M$ with the following property: $t=k-d / 2+1$ is the smallest integer such that every $(t-1)$-space of $\mathrm{PG}(n-1, q)$ is contained in at most one $(k-1)$-space of $\mathscr{C}$. Equivalently, any two distinct codewords of $\mathscr{C}$ intersect in at most a $(t-2)$-space. Hence, $(k-1)$-spaces of $\mathscr{C}$ through a $(t-2)$-space $\Delta$ form a partial $(k-t)$-spread $\mathscr{C}_{\Delta}$ in the quotient geometry PG $(n-t, q)_{\Delta}$ of $\Delta$. Note that $\mathscr{C}_{\Delta}$ is an $\left(n-k+d / 2, M^{\prime}, d, d / 2\right)$-code, called the quotient code of $\Delta$. Using this observation, the following upper bound on the size of a constant dimension code was obtained. This upper bound is known under the name Johnson bound.

Theorem 4 (Johnson bound). [20] The maximal size $\mathscr{A}_{q}(n, d, k)$ of an $(n, M, d, k)$ code satisfies the upper bound

$$
\mathscr{A}_{q}(n, d, k) \leq \frac{\left[\begin{array}{c}
n \\
t-1
\end{array}\right]_{q}}{\left[\begin{array}{c}
k \\
t-1
\end{array}\right]_{q}} \mathscr{A}_{q}(n-k+d / 2, d, d / 2),
$$


where $t=k-d / 2+1$ and where $\mathscr{A}_{q}(n-k+d / 2, d, d / 2)$ is the maximal size of $a$ partial $(d / 2-1)$-spread in $\mathrm{PG}(n-k+d / 2-1, q)$.

Corollary 1. The maximal size $\mathscr{A}_{q}(n, d, k)$ of an $(n, M, d, k)$-code, with $n-k+$ $d / 2 \equiv 0(\bmod d / 2)$, satisfies the upper bound

$$
\mathscr{A}_{q}(n, d, k) \leq \frac{\left[\begin{array}{l}
n \\
t
\end{array}\right]_{q}}{\left[\begin{array}{l}
k \\
t
\end{array}\right]_{q}} .
$$

The main problem is whether there exist constant dimension codes meeting the Johnson bound.

For $d=2 k$, this is the classical partial spreads problem, mentioned above. This shows that a particular problem on subspace codes is completely equivalent to a geometrical problem, already for decades of interest and of importance in Galois geometries. This is one of the classical examples of the close links between the theory of subspace codes and the theory of Galois geometries.

For more detailed information on partial spreads in finite projective spaces, we refer to Chapter 7 of this Springer special volume.

\subsection{Subspace codes from maximum rank distance codes}

Rank distance codes were introduced by Delsarte [14] and are suitable for error correction in the case where the network topology and the underlying network code are known (the coherent case).

The set $\mathscr{M}_{m \times n}(q)$ of $m \times n$ matrices over the finite field $\mathbb{F}_{q}$ forms a metric space with respect to the rank distance, defined by

$$
d_{r}(A, B)=r k(A-B) .
$$

The maximum size of a code of minimum distance $d, 1 \leq d \leq \min \{m, n\}$, in $\left(\mathscr{M}_{m \times n}(q), d_{r}\right)$ is $q^{n(m-d+1)}$ for $m \leq n$ and $q^{m(n-d+1)}$ for $m \geq n$. A code $\mathscr{A} \subset$ $\mathscr{M}_{m \times n}(q)$ attaining this bound is said to be an $(m, n, k)_{q}$ maximum rank distance code (MRD code), where $k=m-d+1$ for $m \leq n$ and $k=n-d+1$ for $m \geq n$. A rank distance code $\mathscr{A}$ is called $\mathbb{F}_{q}$-linear if $\mathscr{A}$ is a subspace of $\mathscr{M}_{m \times n}(q)$ considered as a vector space over $\mathbb{F}_{q}$. We can always assume that $m \leq n$.

In [40], the authors introduced a method, called the lifting process, to construct a constant dimension subspace code from a maximum rank distance code. Let $A$ be an $m \times n$ matrix over $\mathbb{F}_{q}$, and let $I_{m}$ be the $m \times m$ identity matrix. The rows of the $m \times(n+m)$ matrix $\left(I_{m} \mid A\right)$ can be viewed as coordinates of points in general position of an $(m-1)$-dimensional projective space of $\mathrm{PG}(n+m-1, q)$. This subspace is denoted by $L(A)$. Hence, the matrix $A$ can be lifted to a subspace $L(A)$. Let $\mathscr{C}$ be an $(m, n, k)_{q}$ MRD code, and let $A_{1}$ and $A_{2}$ be two distinct matrices of $\mathscr{C}$. Since $r k\left(A_{1}-A_{2}\right) \geq d$, we have that 


$$
r k\left(\begin{array}{ll}
I_{m} & A_{1} \\
I_{m} & A_{2}
\end{array}\right)=m+r k\left(A_{1}-A_{2}\right) \geq m+d .
$$

Hence, $L\left(A_{1}\right)$ meets $L\left(A_{2}\right)$ in at most an $(m-d-1)$-dimensional projective space and $\{L(A) \mid A \in \mathscr{C}\}$ is an $\left(n+m, q^{n k}, 2 d, m\right)$ subspace code. A constant dimension subspace code such that all of its codewords are lifted codewords of an MRD code is called a lifted MRD code.

This lifting process implies the following result.

Theorem 5. [40]

$$
\mathscr{A}_{q}(n+m, 2 d, m) \geq q^{n(m-d+1)} .
$$

Although the size of a lifted MRD code equals the highest power of $q$ in the Johnson bound, it is known that it is not maximal and it can be extended to a larger subspace code.

Several examples of linear $(n, n, k)_{q}$ MRD codes are known to exist (Gabidulin codes [14, 22], twisted Gabidulin codes [39], generalized twisted Gabidulin codes [36]).

\subsection{Planes in PG $(5, q)$ pairwise intersecting in at most a point}

Let us consider an other case for the Johnson bound.

Honold, Kiermaier, and Kurz proved that $\mathscr{A}_{2}(6,4,3)=77$ and there are exactly five codes of size 77 [27].

Geometrically, this means that, in the projective space $\mathrm{PG}(5,2)$, a set of projective planes pairwise intersecting in at most one projective point, has size at most 77, and there are exactly five different examples of such sets of 77 planes.

Honold, Kiermaier, and Kurz managed to extend one of the five examples to an infinite class of $q^{6}+2 q^{2}+2 q+1$ planes in $\operatorname{PG}(5, q)$, pairwise intersecting in at most one point.

Alternatively, Cossidente and Pavese [11] also constructed an infinite class of $q^{6}+2 q^{2}+2 q+1$ planes in $\operatorname{PG}(5, q)$, pairwise intersecting in at most one point. We wish to highlight this construction since it relies on many nice geometrical properties and results. This includes bundles of conics in a plane of $\mathrm{PG}(3, q)$, hyperbolic quadrics in $\mathrm{PG}(3, q)$, and the Klein correspondence between the lines of $\operatorname{PG}(3, q)$ and the Klein quadric $Q^{+}(5, q)$ of $\mathrm{PG}(5, q)$.

We now present the construction of a set of $q^{6}+2 q^{2}+2 q+1$ planes in $\operatorname{PG}(5, q)$, pairwise intersecting in at most one point, by Cossidente and Pavese. This leads to the result

\section{Theorem 6.}

$$
q^{6}+2 q^{2}+2 q+1 \leq \mathscr{A}_{q}(6,4,3) \leq\left(q^{3}+1\right)^{2} .
$$

The construction of Cossidente and Pavese uses the Klein correspondence between lines of $\mathrm{PG}(3, q)$ and their Plücker coordinates on the Klein quadric $Q^{+}(5, q)$ in $\operatorname{PG}(5, q)$ [25, Section 15.4]. 
We first present the hyperbolic quadric $Q^{+}(3, q)$ of $\operatorname{PG}(3, q)$. This is the quadric with standard equation $Q^{+}(3, q): X_{0} X_{2}-X_{1} X_{3}=0$.

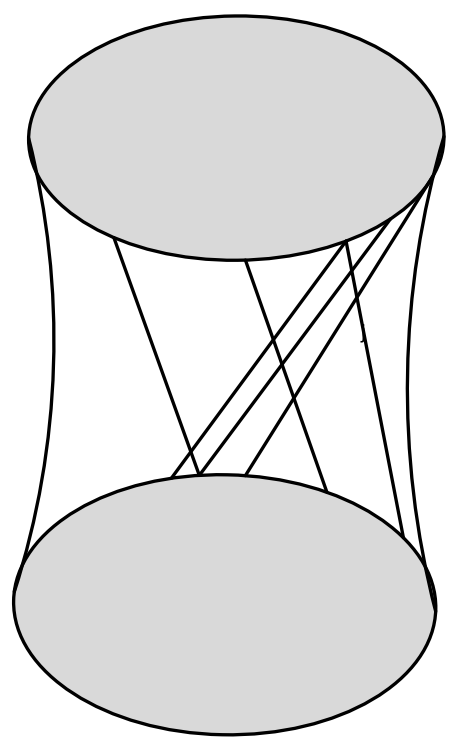

Figure 1: The hyperbolic quadric $Q^{+}(3, q)$

This quadric contains two particular sets of $q+1$ lines, which play a symmetrical role, and which are called a regulus $\mathscr{R}$ and its opposite regulus $\mathscr{R}^{\perp}$.

Lines of a regulus are pairwise disjoint, while the lines of one regulus intersect the lines of the opposite regulus in one point. Figure 1 presents a drawing of a 3dimensional hyperbolic quadric, with three lines of every regulus.

We now introduce the basic facts of the Klein correspondence which plays a central role in the construction of Cossidente and Pavese [25].

Definition 2. Consider a line $\ell$ in $\operatorname{PG}(3, q)$ and suppose that $y\left(y_{0}, y_{1}, y_{2}, y_{3}\right)$ and $z\left(z_{0}, z_{1}, z_{2}, z_{3}\right)$ are different points of $\ell$. Let $p_{i j}=y_{i} z_{j}-y_{j} z_{i}$.

Then the Plücker coordinates of the line $\ell$ are the 6-tuple $\left(p_{01}, p_{02}, p_{03}, p_{23}, p_{31}, p_{12}\right)$.

The Plücker coordinates of a line $\ell$ in $\mathrm{PG}(3, q)$ are well-defined. However, not every 6-tuple is Plücker coordinates of a line $\ell$ of $\mathrm{PG}(3, q)$.

Theorem 7. The 6-tuple $\left(p_{01}, p_{02}, p_{03}, p_{23}, p_{31}, p_{12}\right)$ is Plücker coordinates of a line $\ell$ of $\mathrm{PG}(3, q)$ if and only if

$$
p_{01} p_{23}+p_{02} p_{31}+p_{03} p_{12}=0 .
$$


The set of points $\mathscr{Q}$ of $\operatorname{PG}(5, q)$, defined by the equation $p_{01} p_{23}+p_{02} p_{31}+$ $p_{03} p_{12}=0$, is a hyperbolic quadric $Q^{+}(5, q)$ of $\operatorname{PG}(5, q)$, called the Klein quadric. This hyperbolic quadric $Q^{+}(5, q)$ contains points, lines and planes. The planes of the hyperbolic quadric $Q^{+}(5, q)$ are also called the generators of $Q^{+}(5, q)$. The link with the corresponding sets of lines in $\operatorname{PG}(3, q)$ is defined in the following list [25, Section 15.4].

- Point of $\mathrm{PG}(5, q)$ on the Klein quadric defines a line $\ell$ of $\mathrm{PG}(3, q)$.

- Line of $\operatorname{PG}(5, q)$ on the Klein quadric defines the set of $q+1$ lines of $\operatorname{PG}(3, q)$ through a fixed point $P$ in a fixed plane $\pi$.

- Planes of $\operatorname{PG}(5, q)$ on the Klein quadric define either the set of all lines of $\operatorname{PG}(3, q)$ through a fixed point $P$, or the set of all lines of $\mathrm{PG}(3, q)$ lying in a fixed plane $\pi$.

A Greek plane is a plane contained in the Klein quadric, defining the lines of $\mathrm{PG}(3, q)$ lying in a fixed plane $\pi$, and a Latin plane is a plane contained in the Klein quadric, defining the lines of $\mathrm{PG}(3, q)$ through a fixed point $P$.

- Greek planes, respectively Latin planes, on the Klein quadric pairwise intersect in one point, while a Latin and a Greek plane either are skew to each other, or intersect in a line.

- Consider a plane $\pi$ of $\operatorname{PG}(5, q)$, intersecting the Klein quadric in a conic $C$. This conic corresponds to a regulus $\mathscr{R}$ of a hyperbolic quadric $Q^{+}(3, q)$ in $\operatorname{PG}(3, q)$. The polar plane $\pi^{\perp}$ of $\pi$ with respect to the Klein quadric also intersects the Klein quadric in a conic $C^{\perp}$, corresponding to the opposite regulus $\mathscr{R}^{\perp}$ of $\mathscr{R}$ of this hyperbolic quadric $Q^{+}(3, q)$ in $\mathrm{PG}(3, q)$.

These two polar planes $\pi$ and $\pi^{\perp}$ are skew to each other in $\operatorname{PG}(5, q)$.

In the preceding description, we recognize already the fact that the set of planes of the Klein quadric can be partitioned into two equivalence classes. Two planes of the Klein quadric are called equivalent when they are equal or intersect in a point [26, p. 20]. This definition leads to an equivalence relation on the set of planes of the Klein quadric, having two distinct equivalence classes. They are in the preceding description respectively the class of the Greek planes and the class of the Latin planes. The equivalence classes of the generators of the Klein quadric are also sometimes called the systems of generators.

The construction of Cossidente and Pavese of a set of $q^{6}+2 q^{2}+2 q+1$ planes of $\operatorname{PG}(5, q)$, pairwise intersecting in at most one point, starts by considering specific structures in $\mathrm{PG}(3, q)$, and then transferring, via the Klein correspondence, to planes in $\mathrm{PG}(5, q)$.

- Consider a fixed plane $\pi$ of $\mathrm{PG}(3, q)$.

- A bundle of conics $\mathscr{B}$ in this plane $\pi$ is a set of $q^{2}+q+1$ conics pairwise intersecting in one point.

- Each conic of the bundle $\mathscr{B}$ is contained in $q^{3}(q-1) / 2$ hyperbolic quadrics $Q^{+}(3, q)$ of $\mathrm{PG}(3, q)$. 


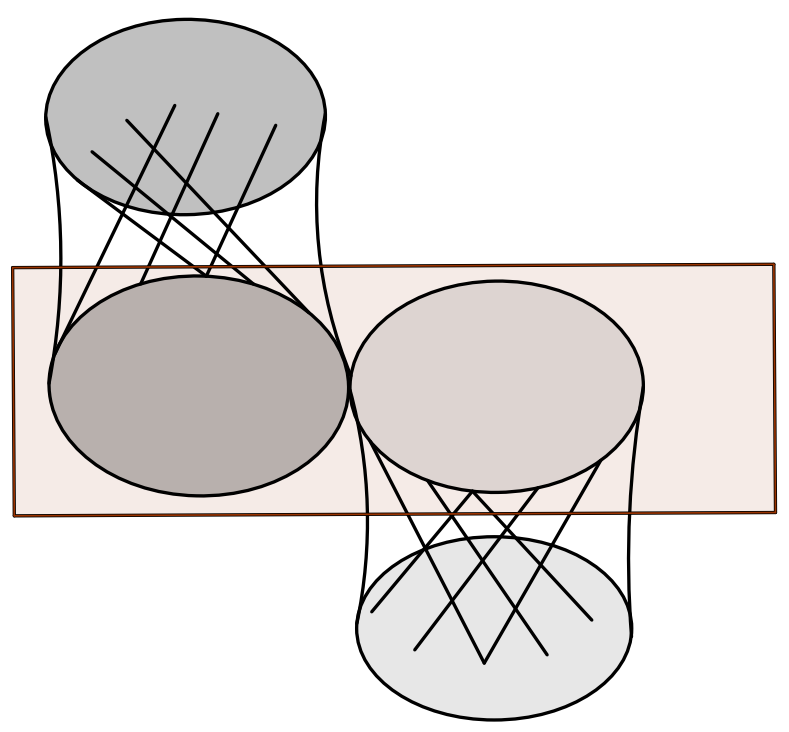

Figure 2: The bundle of conics and the hyperbolic quadrics passing through the conics of the bundle

This leads to $\left(q^{6}-q^{3}\right) / 2$ hyperbolic quadrics $Q^{+}(3, q)$ having $q^{6}-q^{3}$ reguli, and, by applying the Klein correspondence, to a set $\mathscr{L}_{1}$ of $q^{6}-q^{3}$ planes of $\operatorname{PG}(5, q)$, pairwise intersecting in at most one point.

Figure 2 shows a drawing of the plane $\pi$, together with two conics of the bundle of conics $\mathscr{B}$ in this plane $\pi$, and one hyperbolic quadric passing through each one of these two conics.

In the second step of the construction of Cossidente and Pavese, consider this fixed plane $\pi$ in $\operatorname{PG}(3, q)$, containing the bundle of conics $\mathscr{B}$. This plane $\pi$ defines a Greek plane $\Pi$ on the Klein quadric.

Add the other $q^{3}+q^{2}+q$ Greek planes on the Klein quadric to the set $\mathscr{L}_{1}$. This leads to a larger set $\mathscr{L}_{2}$ of $q^{6}+q^{2}+q$ planes of $\mathrm{PG}(5, q)$, pairwise intersecting in at most one point.

In the third step, consider again the Greek plane $\Pi$ in $\mathrm{PG}(5, q)$, corresponding to the plane $\pi$ in $\mathrm{PG}(3, q)$, containing the bundle of conics $\mathscr{B}$.

Each line $r$ of $\Pi$ lies in $q-1$ planes of $\operatorname{PG}(5, q)$ only intersecting the Klein quadric in the line $r$.

For every line $r$ of $\Pi$, select one such plane, and add it to the set $\mathscr{L}_{2}$. This gives the desired set $\mathscr{L}$ of $q^{6}+2 q^{2}+2 q+1$ planes of $\mathrm{PG}(5, q)$, pairwise intersecting in at most one point. 


\subsection{Planes in $\mathrm{PG}(5, q)$ pairwise intersecting in at most a point (Alternative construction)}

Let $U_{i}=(0, \ldots, 0,1,0, \ldots, 0), i=0, \ldots, 5$, be the vector with the one in the $(i+1)$-th position.

Let $\mathscr{C}$ be a linear $(3,3,2)_{q}$ MRD code. Let $\mathscr{L}=\{L(A) \mid A \in \mathscr{C}\}$ be the lifted MRD code obtained by lifting the elements of $\mathscr{C}$. Then $\mathscr{L}$ consists of $q^{6}$ planes of $\operatorname{PG}(5, q)$ mutually intersecting in at most a point. In particular, members of $\mathscr{L}$ are disjoint from the special plane $T=\left\langle U_{3}, U_{4}, U_{5}\right\rangle$ and therefore every line covered by an element of $\mathscr{L}$ is disjoint from $T$. Moreover, from [27, Lemma 6], every line of $\operatorname{PG}(5, q)$ disjoint from $T$ is covered by a member of $\mathscr{L}$ exactly once. We denote by $T^{\prime}$ the plane $\left\langle U_{0}, U_{1}, U_{2}\right\rangle$. Since the zero matrix belongs to $\mathscr{C}$, we have that $T^{\prime} \in \mathscr{L}$.

Let $\mathscr{S}$ denote the set of $q^{3} 3 \times 3$ skew-symmetric matrices over $\mathbb{F}_{q}$. Here, for $q$ even, the diagonal elements of a $3 \times 3$-skew symmetric matrix are zeros. Since there exists a linear $(3,3,2)_{q}$ MRD code containing $\mathscr{S}$, we may assume that $\mathscr{S} \subset \mathscr{C}$, see [12, Proposition 3.1].

Now, we introduce the non-degenerate hyperbolic quadric $\mathscr{Q}$ of $\operatorname{PG}(5, q)$ having the following equation:

$$
X_{0} X_{3}+X_{1} X_{4}+X_{2} X_{5}=0 .
$$

The planes $T$ and $T^{\prime}$ are generators of $\mathscr{Q}$. They belong to different systems of generators of $\mathscr{Q}$. Let $\mathscr{M}, \mathscr{M}^{\prime}$ be the system of generators of $\mathscr{Q}$ containing $T, T^{\prime}$, respectively.

It can be seen that if $A$ is a $3 \times 3$ skew-symmetric matrix over $\mathbb{F}_{q}$, then $L(A)$ is a generator of $\mathscr{Q}$ disjoint from $T$.

Remark 1. Since the number of generators of $\mathscr{Q}$ disjoint from $T$ equals $q^{3}$, we have that each such a plane is of the form $L(A)$, for some $A \in \mathscr{S}$. Note that, if $A \in \mathscr{S}$, then $L(A)$ belongs to the system $\mathscr{M}^{\prime}$ of generators containing $T^{\prime}$.

Let $\mathscr{L}^{\prime}$ be the set consisting of the $q^{3}$ planes obtained by lifting the matrices of $\mathscr{S}$. Then, the set $\left(\mathscr{L} \backslash \mathscr{L}^{\prime}\right) \cup \mathscr{M}$ consists of $q^{6}+q^{2}+q+1$ planes mutually intersecting in at most a point.

Corresponding to a line $r$ of $T$, there corresponds a set $\mathscr{T}_{r}$ of $q-1$ planes of $\operatorname{PG}(5, q)$ meeting $\mathscr{Q}$ exactly in $r$. Varying the line $r$ over the line set of $T$ and choosing one of the planes in $\mathscr{T}_{r}$, we obtain a set $\mathscr{T}$ of $q^{2}+q+1$ planes mutually intersecting in at most one point.

Finally, we have that the set $\left(\mathscr{L} \backslash \mathscr{L}^{\prime}\right) \cup \mathscr{M} \cup \mathscr{T}$ is a set of $q^{6}+2 q^{2}+2 q+1$ planes mutually intersecting in at most a point.

\subsection{Solids in $\mathrm{PG}(7, q)$ pairwise intersecting in at most a line}

Let $\mathscr{C}$ be a linear $(4,4,3)_{q}$ MRD code. Let $\mathscr{L}_{1}=\{L(A) \mid A \in \mathscr{C}\}$ be the lifted MRD code obtained by lifting the elements of $\mathscr{C}$. Then $\mathscr{L}_{1}$ consists of $q^{12}$ solids 
of $\operatorname{PG}(7, q)$ mutually intersecting in at most a line. In particular, members of $\mathscr{L}_{1}$ are disjoint from the special solid $T=\left\langle U_{4}, U_{5}, U_{6}, U_{7}\right\rangle$ and therefore every plane covered by an element of $\mathscr{L}_{1}$ is disjoint from $T$. Moreover, from [27, Lemma 6], every plane of $\operatorname{PG}(7, q)$ disjoint from $T$ is covered by a member of $\mathscr{L}_{1}$ exactly once. We denote by $T^{\prime}$ the solid $\left\langle U_{0}, U_{1}, U_{2}, U_{3}\right\rangle$. Since the zero matrix belongs to $\mathscr{C}$, we have that $T^{\prime} \in \mathscr{L}_{1}$.

Let $\mathscr{C}_{r} \subset \mathscr{C}$ be the set consisting of all the matrices of $\mathscr{C}$ having rank $r$, with $2 \leq$ $r \leq 4$. From [22], it is known that a linear $(4,4,3)_{q}$ MRD code contains $\left(q^{4}-1\right)\left(q^{2}+\right.$ 1) $\left(q^{2}+q+1\right)$ matrices of rank $2,\left(q^{4}-1\right)\left(q^{2}+1\right)(q+1)\left(q^{4}-q^{2}-q\right)$ matrices of rank 3 , and $\left(q^{4}-1\right) q^{3}\left(q^{5}-q^{4}-q^{3}+q+1\right)$ matrices of rank 4 .

Let $A$ be an element of $\mathscr{C}_{2}$. As in Section 3.2, the rows of the $4 \times 8$ matrix $\left(A \mid I_{4}\right)$ can be viewed as coordinates of points in general position of a solid, say $L^{\prime}(A)$, of $\operatorname{PG}(7, q)$. The solid $L^{\prime}(A)$ is disjoint from $T^{\prime}$ and meets $T$ in a line. Let $\mathscr{L}_{2}=$ $\left\{L^{\prime}(A) \mid A \in \mathscr{C}_{2}\right\}$ be the set of solids obtained from the elements of $\mathscr{C}_{2}$. Then we have that $\mathscr{L}_{1} \cup \mathscr{L}_{2}$ is a set of $q^{12}+\left(q^{4}-1\right)\left(q^{2}+1\right)\left(q^{2}+q+1\right)$ solids of $\operatorname{PG}(7, q)$ mutually intersecting in at most a line.

Let $\mathscr{S}$ denote the set of $q^{6} 4 \times 4$ skew-symmetric matrices over $\mathbb{F}_{q}$. Here, for $q$ even, the diagonal elements of a $4 \times 4$-skew symmetric matrix are zeros. Since there exists a linear $(4,4,3)_{q}$ MRD code containing $\mathscr{S}$, we may assume that $\mathscr{S} \subset \mathscr{C}$, see [12, Proposition 3.1].

Now, we introduce the non-degenerate hyperbolic quadric $\mathscr{Q}_{1}$ of $\operatorname{PG}(7, q)$ having the following equation:

$$
X_{0} X_{4}+X_{1} X_{5}+X_{2} X_{6}+X_{3} X_{7}=0 .
$$

Here again, the solids (generators) of the hyperbolic quadric $\mathscr{Q}_{1}$ of $\operatorname{PG}(7, q)$ are partitioned into two equivalence classes $\mathscr{M}_{1}$ and $\mathscr{M}_{1}^{\prime}$ [26]. Two generators $\Pi_{1}$ and $\Pi_{2}$ are called equivalent when they are equal or intersect in a line. This relation is again an equivalence relation, having two equivalence classes $\mathscr{M}_{1}$ and $\mathscr{M}_{1}^{\prime}$ on the set of generators of the hyperbolic quadric $\mathscr{Q}_{1}$ of $\operatorname{PG}(7, q)$.

The solids $T$ and $T^{\prime}$ are generators of $\mathscr{Q}_{1}$. They belong to the same system of generators of $\mathscr{Q}_{1}$, say $\mathscr{M}_{1}$. Let $D(X)$ and $I(X)$ denote the set of generators in $\mathscr{M}_{1}$ disjoint from the solid $X$ or meeting non-trivially $X$, respectively. Then

$$
\mathscr{M}_{1}=D(T) \cup\left(D\left(T^{\prime}\right) \cap I(T)\right) \cup\left(I\left(T^{\prime}\right) \cap I(T)\right),
$$

where $D(T), D\left(T^{\prime}\right) \cap I(T)$ and $I\left(T^{\prime}\right) \cap I(T)$ are trivially intersecting sets.

It can be seen that if $A$ is a $4 \times 4$ skew-symmetric matrix over $\mathbb{F}_{q}$, then $L(A)$ (resp. $L^{\prime}(A)$ ) is a generator of $\mathscr{Q}_{1}$ disjoint from $T$ (resp. $T^{\prime}$ ).

Remark 2. Since the number of generators of $\mathscr{Q}_{1}$ disjoint from $T$ equals $q^{6}$ [30, Lemma 3], we have that each such a solid is of the form $L(A)$, for some $A \in \mathscr{S}$. Note that, if $A \in \mathscr{S}$, then $L(A)$ belongs to $\mathscr{M}_{1}$.

On the other hand, a solid $L^{\prime}(A)$ in $D\left(T^{\prime}\right)$ is disjoint from $T$ if and only if $A$ is a skew-symmetric matrix of rank 4 . Therefore, the number of skew-symmetric matrices of rank 4 is equal to $\left|D(T) \cap D\left(T^{\prime}\right)\right|$. It follows that 


$$
\begin{gathered}
\left|D\left(T^{\prime}\right) \cap I(T)\right|=\left|D\left(T^{\prime}\right)\right|-\left|D\left(T^{\prime}\right) \cap D(T)\right|= \\
\quad=q^{6}-(q-1) q^{2}\left(q^{3}-1\right)=q^{2}\left(q^{3}+q-1\right)
\end{gathered}
$$

and

$$
\left|I\left(T^{\prime}\right) \cap I(T)\right|=\left|\mathscr{M}_{1}\right|-2 q^{6}+(q-1) q^{2}\left(q^{3}-1\right)=\left(q^{2}+1\right)\left(q^{2}+q+1\right) .
$$

Since $\mathscr{S} \subset \mathscr{C}$, we have that $D(T) \subset \mathscr{L}_{1}$. Moreover, every element $g \in D\left(T^{\prime}\right) \cap$ $I(T)$ is of the form $L^{\prime}(A)$ for some skew-symmetric matrix $A$ having rank 2. Hence, $g \in \mathscr{L}_{2}$. The set $\mathscr{L}_{1} \cup \mathscr{L}_{2} \cup\left(I\left(T^{\prime}\right) \cap I(T)\right)$ consists of $q^{12}+\left(q^{4}-1\right)\left(q^{2}+1\right)\left(q^{2}+\right.$ $q+1)+\left(q^{2}+1\right)\left(q^{2}+q+1\right)$ solids of $\mathrm{PG}(7, q)$ mutually intersecting in at most a line.

Let $\gamma$ be a non-zero element of $\mathbb{F}_{q}$ such that the polynomial $X^{2}-X-\gamma$ is irreducible over $\mathbb{F}_{q}$. Let $\mathscr{Q}_{2}$ be the hyperbolic quadric of $\operatorname{PG}(7, q)$ having equation

$$
X_{0} X_{6}+X_{1} X_{7}+\gamma^{-1}\left(X_{2} X_{4}+X_{3} X_{5}+X_{2} X_{6}+X_{3} X_{7}\right)=0 .
$$

The hyperbolic quadrics $\mathscr{Q}_{1}$ and $\mathscr{Q}_{2}$ generate a pencil of hyperbolic quadrics of $\operatorname{PG}(7, q)$, say $\mathscr{F}$, containing $q-1$ other distinct quadrics, say $\mathscr{Q}_{i}, 3 \leq i \leq q+1$, none of which is degenerate. Let $\mathscr{X}$ be the base locus of $\mathscr{F}$. Since the hyperbolic quadrics of $\mathscr{F}$ cover all the points of $\operatorname{PG}(7, q)$, and any two distinct quadrics in $\mathscr{F}$ intersect precisely in $\mathscr{X}$, we have that $|\mathscr{X}|=(q+1)\left(q^{2}+1\right)^{2}$. There are $2\left(q^{2}+1\right)$ generators belonging to each hyperbolic quadric of the pencil $\mathscr{F}$ and they all belong to the same system of generators with respect to each of the quadrics $\mathscr{Q}_{i}$ in $\mathscr{F}$, say $\mathscr{M}_{i}$. In particular, $T$ and $T^{\prime}$ belong to each hyperbolic quadric of the pencil $\mathscr{F}$. Let $\mathscr{G}$ be the set of generators meeting both $T$ and $T^{\prime}$ non-trivially, and belonging to each hyperbolic quadric of the pencil $\mathscr{F}$. We have that $\mathscr{G} \subset \mathscr{M}_{i}$, for every $1 \leq i \leq q+1$, and $|\mathscr{G}|=q^{2}+1$.

Let $I_{i}(X)$ denote the set of solids in $\mathscr{M}_{i}$ meeting non-trivially $X, 2 \leq i \leq q+1$. Then $\mathscr{G}=\bigcap_{i=2}^{q+1}\left(I_{i}(T) \cap I_{i}\left(T^{\prime}\right)\right) \cap\left(I(T) \cap I\left(T^{\prime}\right)\right)$.

The set $\mathscr{L}_{1} \cup \mathscr{L}_{2} \cup\left(\bigcup_{i=2}^{q+1}\left(I_{i}(T) \cap I_{i}\left(T^{\prime}\right)\right)\right) \cup\left(I(T) \cap I\left(T^{\prime}\right)\right)$ is a set of $q^{12}+\left(q^{4}-\right.$ 1) $\left(q^{2}+1\right)\left(q^{2}+q+1\right)+q(q+1)^{2}\left(q^{2}+1\right)+\left(q^{2}+1\right)$ solids of $\mathrm{PG}(7, q)$ mutually intersecting in at most a line.

The set $\mathscr{G}$ consists of $q^{2}+1$ generators belonging to each hyperbolic quadric of the pencil $\mathscr{F}$ such that every element in $\mathscr{G}$ meets both $T$ and $T^{\prime}$ in a line. The set $\mathscr{D}_{T}=\{A \cap T \mid A \in \mathscr{G}\}, \mathscr{D}_{T^{\prime}}=\left\{A \cap T^{\prime} \mid A \in \mathscr{G}\right\}$ is a line-spread of $T, T^{\prime}$, respectively. In particular, for a fixed line $\ell \in \mathscr{D}_{T}$, there exists a unique element in $\mathscr{D}_{T^{\prime}}$, say $A_{\ell}$, such that $\left\langle\ell, A_{\ell}\right\rangle$ is in $\mathscr{G}$, and viceversa. Furthermore, if $\ell \in \mathscr{D}_{T}$ and $B \in \mathscr{D}_{T^{\prime}} \backslash\left\{A_{\ell}\right\}$, then $\langle\ell, B\rangle$ is a solid meeting a hyperbolic quadric of the pencil $\mathscr{F}$ in a 3-dimensional hyperbolic quadric $\mathscr{Q}^{+}(3, q)$. Let $\mathscr{D}^{\prime}$ be the set of solids of the form $\langle\ell, B\rangle$, where $\ell \in \mathscr{D}_{T}$ and $B \in \mathscr{D}_{T^{\prime}} \backslash\left\{A_{\ell}\right\}$. Then $\mathscr{D}^{\prime}$ is disjoint from $\mathscr{G}$ and $\left|\mathscr{D}^{\prime}\right|=q^{2}\left(q^{2}+1\right)$. We have that $\mathscr{L}_{1} \cup \mathscr{L}_{2} \cup\left(\bigcup_{i=2}^{q+1}\left(I_{i}(T) \cap I_{i}\left(T^{\prime}\right)\right)\right) \cup\left(I(T) \cap I\left(T^{\prime}\right)\right) \cup$ $\mathscr{D}^{\prime} \cup\{T\}$ is a set of solids mutually intersecting in at most a line, of size

$$
q^{12}+\left(q^{4}-1\right)\left(q^{2}+1\right)\left(q^{2}+q+1\right)+\left(q^{3}+3 q^{2}+q+1\right)\left(q^{2}+1\right)+1 .
$$


There exists a group $H$ in the orthogonal group $\mathrm{PGO}^{+}(8, q)$, stabilizing $\mathscr{Q}_{1}$, fixing both $T, T^{\prime}$, their line-spreads $\mathscr{D}(T), \mathscr{D}\left(T^{\prime}\right)$, and permuting in a single orbit the remaining lines of $T$ (respectively $T^{\prime}$ ). Let $\perp$ be the orthogonal polarity of $\operatorname{PG}(7, q)$ associated with $\mathscr{Q}_{1}$. If $r^{\prime}$ is a line of $T^{\prime}$, then $r^{\perp}$ meets $T$ in a line $r$. If $r^{\prime}$ belongs to $\mathscr{D}_{T^{\prime}}$, then $r$ belongs to $\mathscr{D}_{T}$. Assume that $r^{\prime}$ does not belong to $\mathscr{D}_{T^{\prime}}$. Of course, $r^{\prime}$ meets $q+1$ lines $l_{1}^{\prime}, \ldots, l_{q+1}^{\prime}$ of $\mathscr{D}_{T^{\prime}}$ and $r$ meets $q+1$ lines $l_{1}, \ldots, l_{q+1}$ of $\mathscr{D}_{T}$.

The group $H$ contains a subgroup fixing the lines $l_{1}^{\prime}, \ldots, l_{q+1}^{\prime}$ and having $q(q-$ 1) $/ 2$ orbits of size $q^{2}-q$ on the lines of $T$ distinct from $l_{1}, \ldots, l_{q+1}$. Each one of them, together with $l_{1}, \ldots, l_{q+1}$, is a line-spread of $T$, one of them being $\mathscr{D}_{T}$. Let $\mathscr{E}$ be one of the orbits of size $q^{2}-q$ disjoint from $\mathscr{D}_{T}$ and let $Y$ be the solid generated by $r^{\prime}$ and a line of $\mathscr{E}$. It is possible to prove that $Y^{H}$ is a set of $q^{6}-q^{2}$ solids mutually intersecting in at most a line.

Finally, we have that the set $\mathscr{L}_{1} \cup \mathscr{L}_{2} \cup\left(\bigcup_{i=2}^{q+1}\left(I_{i}(T) \cap I_{i}\left(T^{\prime}\right)\right)\right) \cup\left(I(T) \cap I\left(T^{\prime}\right)\right) \cup$ $\mathscr{D}^{\prime} \cup Y^{H} \cup\{T\}$ is an $(8, M, 4,4)_{q}$-subspace code, where

$$
M=q^{12}+q^{2}\left(q^{2}+1\right)^{2}\left(q^{2}+q+1\right)+1 .
$$

This leads to the following result.

\section{Corollary 2.}

$$
\mathscr{A}_{q}(8,4,4) \geq q^{12}+q^{2}\left(q^{2}+1\right)^{2}\left(q^{2}+q+1\right)+1 .
$$

Remark 3. The previous lower bound was obtained with different techniques in [19], where the authors, among other interesting results, proved that $q^{12}+q^{2}\left(q^{2}+\right.$ $1)^{2}\left(q^{2}+q+1\right)+1$ is also the maximum size of an $(8, M, 4,4)_{q}$-subspace code containing a lifted MRD code.

For more information on constant dimension codes, we also refer to Chapter 2 of this Springer special volume.

\section{Optimal mixed-dimension subspace codes in $\operatorname{PG}(4, q)$}

Via geometrical arguments, it can be shown that $\mathscr{A}_{q}(5,3)=2\left(q^{3}+1\right)$. Here, a $\left(5,2\left(q^{3}+1\right), 3\right)$-code consists of subspaces of the vector space $V(5, q)$, equivalently, of subspaces of the projective space $\mathrm{PG}(4, q)$.

To give an idea which arguments are used to get geometrical insight in which subspaces could be contained in a mixed-dimension $(5, M, 3)$-subspace code, we try to see how the codewords of this code can intersect.

The vector space $V(5, q)$ has four types of subspaces: vector lines, vector planes, subspaces of dimension three, and subspaces of dimension four.

The formula for the subspace distance $d\left(U, U^{\prime}\right)=\operatorname{dim}\left(U+U^{\prime}\right)-\operatorname{dim}\left(U \cap U^{\prime}\right)$ shows that a $(5, M, 3)$-subspace code with minimum distance 3 : 
1. cannot contain two vector lines, and cannot contain two subspaces of dimension four,

2. two vector planes in the code should only intersect in the zero vector,

3. two subspaces of dimension three should only intersect in a vector line,

4. a vector line in the code cannot be contained in a vector plane or in a 3dimensional vector space belonging to the code, a vector plane in the code cannot be contained in a 3-dimensional subspace or 4-dimensional subspace belonging to the code, and a 3-dimensional subspace in the code cannot be contained in a 4-dimensional subspace belonging to the code.

We now interpret these conditions in the geometrical setting, so we replace all the codewords in the $\left(5,2\left(q^{3}+1\right), 3\right)$-code by their geometrical equivalents in the projective space $\mathrm{PG}(4, q)$.

Condition (2) implies that two projective lines belonging to the code are skew to each other. Hence, the projective lines belonging to the $\left(5,2\left(q^{3}+1\right), 3\right)$-code form a partial line spread of $\mathrm{PG}(4, q)$.

From Theorem 2, the largest partial line spread of $\mathrm{PG}(4, q)$ has size $q^{3}+1$. So, if $\mathscr{C}$ is an optimal $(5,3)_{q}$ subspace code, then $\mathscr{C}$ contains at most $q^{3}+1$ pairwise skew lines. A dual argument shows that $\mathscr{C}$ contains at most $q^{3}+1$ planes, and these planes pairwise intersect in a projective point.

Hence, if $\mathscr{C}$ consists of projective lines and projective planes, we have that $|\mathscr{C}| \leq$ $2\left(q^{3}+1\right)$ and, if $|\mathscr{C}|=2\left(q^{3}+1\right)$, then $\mathscr{C}$ consists of a set $\mathscr{L}$ of $q^{3}+1$ pairwise skew lines and of a set $\mathscr{P}$ of $q^{3}+1$ planes mutually intersecting in exactly a point, such that no line of $\mathscr{L}$ is contained in a plane of $\mathscr{P}$.

Note that Condition (1) above states that $\mathscr{C}$ contains at most one point and, dually, $\mathscr{C}$ contains at most one solid.

Counting arguments of [13] prove that if $\mathscr{C}$ contains a point, then $\mathscr{C}$ contains at most $q^{3}$ planes. Dually, if $\mathscr{C}$ contains a solid, then $\mathscr{C}$ contains at most $q^{3}$ lines.

It follows from these arguments that $\mathscr{A}_{q}(5,3) \leq 2\left(q^{3}+1\right)$ and there are four possibilities for the code $\mathscr{C}$ :

I) $\mathscr{C}$ consists of one point, $q^{3}+1$ lines, and $q^{3}$ planes;

II) $\mathscr{C}$ consists of $q^{3}$ lines, $q^{3}+1$ planes, and one solid;

III) $\mathscr{C}$ consists of one point, $q^{3}$ lines, $q^{3}$ planes, and one solid;

IV) $\mathscr{C}$ consists of $q^{3}+1$ lines and $q^{3}+1$ planes.

We present the construction for $q$ odd, showing that $\mathscr{A}_{q}(5,3)=2\left(q^{3}+1\right)$. A similar construction exists for $q$ even, but since it is more technical, we refer to [13] for its description.

Let $q=p^{h}$, where $p$ is an odd prime. Let $\mathrm{PG}(4, q)$ be equipped with homogeneous coordinates $\left(X_{0}, X_{1}, X_{2}, X_{3}, X_{4}\right)$, let $\pi$ be the projective plane with equations $X_{3}=X_{4}=0$ and let $\ell$ be the line of $\pi$ with equations $X_{2}=X_{3}=X_{4}=0$. Let $\omega$ be a primitive element of $\mathbb{F}_{q}$ and denote by $\Pi_{i}$ the solid of $\operatorname{PG}(4, q)$ passing through $\pi$ with equation $X_{3}=\omega^{i-1} X_{4}$, if $1 \leq i \leq q-1, X_{3}=0$ if $i=q$, and $X_{4}=0$ if $i=q+1$. 
Let $a, b, c$ be fixed elements of $\mathbb{F}_{q}$ such that the polynomial $X^{3}+a X^{2}+b X+c=0$ is irreducible over $\mathbb{F}_{q}$ and consider the following matrices

$$
M_{r, s, t}=\left(\begin{array}{ccccc}
1 & 0 & r & r^{2}-a r+s & t \\
0 & 1 & s & 2 r s-t & s^{2}+b s-c r \\
0 & 0 & 1 & 2 r & 2 s \\
0 & 0 & 0 & 1 & 0 \\
0 & 0 & 0 & 0 & 1
\end{array}\right) .
$$

Then the group of projective transformations $G=\left\{x \mapsto M_{r, s, t} \cdot x \mid r, s, t \in \mathbb{F}_{q}\right\}$ is a $p$-group of order $q^{3}$.

This group $G$ has within the set of lines of $\mathrm{PG}(4, q)$, exactly $q^{3}$ orbits of size $q^{3}$, each consisting of pairwise disjoint lines that are disjoint from $\pi$. Similarly, every plane of $\mathrm{PG}(4, q)$, intersecting the plane $\pi$ in exactly one point, not belonging to the line $\ell$, belongs to an orbit of $G$, consisting of $q^{3}$ planes, pairwise intersecting in one point.

Consider such an orbit under $G$ of $q^{3}$ planes intersecting the fixed plane $\pi$ in a point not belonging to the line $\ell$. Such a plane contains $q^{2}$ lines skew to $\pi$. They define only $q^{2}$ of the $q^{3}$ orbits of lines that are disjoint from $\pi$. Hence, by taking one of the remaining $q^{3}-q^{2}$ orbits of such lines, a set of $q^{3}$ planes and $q^{3}$ lines is obtained forming a subspace code with minimum distance 3 .

This $\left(5,2 q^{3}, 3\right)$-code can be extended to a $\left(5,2\left(q^{3}+1\right), 3\right)$-code by adding a line $r$ of $\pi, r \neq \ell$, and a plane $\xi$ through the line $\ell$, but with $\xi \neq \pi$, to this code. This leads to an optimal $\left(5, \mathscr{A}_{q}(5,3), 3\right)$-code consisting of $q^{3}+1$ lines and planes.

Figure 3 presents the setting for this mixed dimension code in $\mathrm{PG}(4, q), q$ odd. The drawing shows the plane $\pi$, the line $\ell$ with the plane $\xi$ passing through $\ell$, and the line $r$. Two of the $q+1$ solids through the plane $\pi$ are shown. They are denoted by $\Pi_{i}$ and $\Pi_{j}$. The orbit of $q^{3}$ lines skew to $\pi$ is denoted by the three vertical lines, sharing one point with the solids $\Pi_{i}$ and $\Pi_{j}$. Finally, one plane is drawn of the orbit of $q^{3}$ planes sharing one point with the plane $\pi$, which does not belong to $\ell$, and which are skew to the $q^{3}$ lines of the selected orbit of lines. 


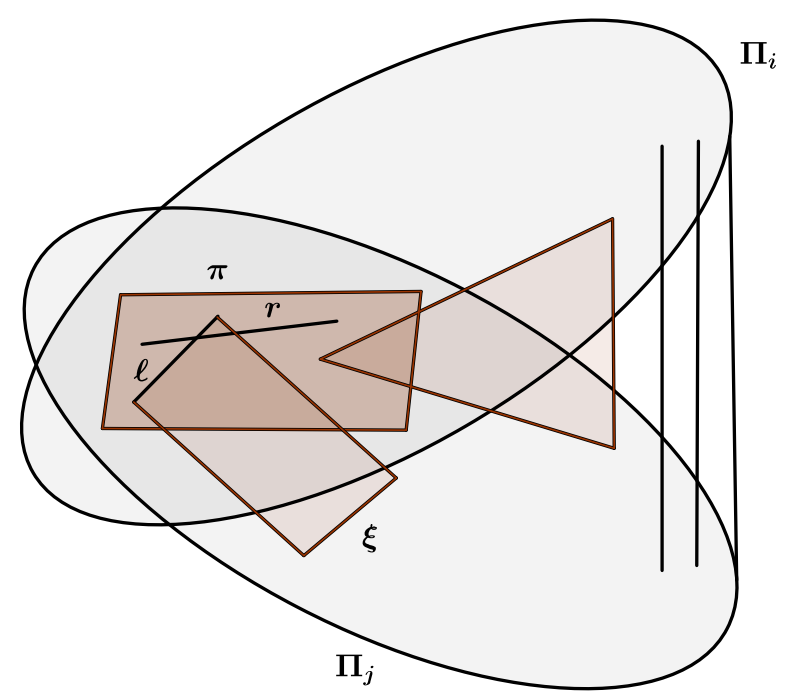

Figure 3: The mixed dimension construction in $\mathrm{PG}(4, q), q$ odd

In [13, Remark 2.6], it is shown that minor changes can be made to the construction, to also construct optimal $\left(5, \mathscr{A}_{q}(5,3), 3\right)$-codes of type I), II), and III).

\section{Geometrical links to non-linear maximum rank distance codes}

In this context, we can make links to the Segre variety of $\mathrm{PG}\left(n^{2}-1, q\right)[10,26]$.

The Segre map

$$
\sigma: \operatorname{PG}(n-1, q) \times \mathrm{PG}(n-1, q) \rightarrow \mathrm{PG}\left(n^{2}-1, q\right),
$$

takes a pair of points $x=\left(x_{0}, \ldots, x_{n-1}\right), y=\left(y_{0}, \ldots, y_{n-1}\right)$ of $\mathrm{PG}(n-1, q)$ to their product $\left(x_{0} y_{0}, x_{0} y_{1}, \ldots, x_{n-1} y_{n-1}\right)$ (the products $x_{i} y_{j}$ are taken in lexicographical order). The image of the Segre map is an algebraic variety of $\operatorname{PG}\left(n^{2}-1, q\right)$, called the Segre variety, and is denoted by $\mathscr{S}_{n-1, n-1}$.

When $n=2$, the Segre variety $\mathscr{S}_{1,1}$ of $\mathrm{PG}(3, q)$ is a non-degenerate hyperbolic quadric $\mathscr{Q}^{+}(3, q)$. In Section 3.3, we defined the hyperbolic quadric as the quadric with equation $X_{0} X_{2}-X_{1} X_{3}=0$, but equivalently, this quadric is given as the zero locus of the quadratic polynomial given by the determinant of the matrix

$$
\left(\begin{array}{ll}
x_{0} y_{0} & x_{0} y_{1} \\
x_{1} y_{0} & x_{1} y_{1}
\end{array}\right)
$$


In the case $n=3$, the Segre variety $\mathscr{S}_{2,2}$ of $\operatorname{PG}(8, q)$ is defined to be the zero locus of all quadratic polynomials given by the determinants of the $2 \times 2$ matrices of the matrix

$$
\left(\begin{array}{lll}
x_{0} y_{0} & x_{0} y_{1} & x_{0} y_{2} \\
x_{1} y_{0} & x_{1} y_{1} & x_{1} y_{2} \\
x_{2} y_{0} & x_{2} y_{1} & x_{2} y_{2}
\end{array}\right) .
$$

In other terms, in the projective space $\operatorname{PG}\left(\mathscr{M}_{n \times n}(q)\right)$, if $n=2$, the Segre variety $\mathscr{S}_{1,1}$ of $\mathrm{PG}(3, q)$ is represented by all $2 \times 2$ matrices of rank 1 and if $n=3$, the Segre variety $\mathscr{S}_{2,2}$ of $\mathrm{PG}(8, q)$ is represented by all $3 \times 3$ matrices of rank 1 .

The following definition considers a set of points that at first sight is of purely geometrical interest with respect to a Segre variety.

Definition 3. An exterior set with respect to a Segre variety $\mathscr{S}_{n-1, n-1}$ of $\operatorname{PG}\left(n^{2}-\right.$ $1, q)$ is a set of points $\mathscr{E}$ of $\mathrm{PG}\left(n^{2}-1, q\right) \backslash \mathscr{S}_{n-1, n-1}$ of size $\left(q^{n^{2}-n}-1\right) /(q-1)$ such that the line joining any two points of $\mathscr{E}$ is disjoint from $\mathscr{S}_{n-1, n-1}$.

But this definition and the previous observations give an immediate link with maximum rank distance codes.

In general, an exterior set $\mathscr{E}$ of $\operatorname{PG}\left(n^{2}-1, q\right)$ with respect to a Segre variety $\mathscr{S}_{n-1, n-1}$, of size $\left(q^{n^{2}-n}-1\right) /(q-1)$, gives rise to a MRD code: this is done by identifying a point of $\mathscr{E}$ and its nonzero scalar multiples together with the zero matrix with members of $\mathscr{M}_{n \times n}(q)$. This is also the key tool of our approach. We formulate this in the next proposition.

Proposition 1. An exterior set with respect to $\mathscr{S}_{n-1, n-1}$ gives rise to an $(n, n, n-1)$ MRD code closed under $\mathbb{F}_{q}-$ multiplication, and viceversa.

Corollary 3. An $(n, n, n-1) \mathbb{F}_{q}$-linear Gabidulin code $\mathscr{G}$ is a certain subspace $X$ of $\operatorname{PG}\left(n^{2}-1, q\right)$ of dimension $n^{2}-n-1$ which is an exterior set with respect to $\mathscr{S}_{n-1, n-1}$.

The preceding corollary is of particular interest since the maximum dimension of a subspace of $\mathrm{PG}\left(n^{2}-1, q\right)$ disjoint from $\mathscr{S}_{n-1, n-1}$ is exactly $n^{2}-n-1$ [9].

\subsection{The case $n=2$}

In this subsection, we report the complete classification of linear and non-linear MRD codes that are closed under $\mathbb{F}_{q}$-multiplication when $n=m=2$. We do this because this is linked to the solution of a purely geometrical problem, related to the hyperbolic quadric $\mathscr{Q}^{+}(3, q)$ of the projective space $\mathrm{PG}(3, q)$, which is the smallest example of a Segre variety.

A flock of the hyperbolic quadric $\mathscr{Q}^{+}(3, q)$ of the finite projective space $\mathrm{PG}(3, q)$ is a partition of $\mathscr{Q}^{+}(3, q)$ consisting of $q+1$ irreducible conics. A linear flock of $\mathscr{Q}^{+}(3, q)$ is a flock which consists of $q+1$ irreducible conics, lying in the $q+1$ 
planes through a fixed line $\ell$ skew to the hyperbolic quadric $\mathscr{Q}^{+}(3, q)$. A maximal exterior set (MES) with respect to the hyperbolic quadric $\mathscr{Q}^{+}(3, q)$ is a set of $q+1$ points of $\mathrm{PG}(3, q)$ such that the line joining any two of them has no point in common with $\mathscr{Q}^{+}(3, q)$. The polar planes, with respect to the polarity induced by $\mathscr{Q}^{+}(3, q)$, of the points of a MES, define a flock, and conversely.

In [41], J.A. Thas proved that all flocks of $\mathscr{Q}^{+}(3, q)$ are linear if $q$ is even, and that $\mathscr{Q}^{+}(3, q)$ has non-linear flocks (called Thas flocks) if $q$ is odd. Furthermore, he showed that, for $q=3,7$ and $q \equiv 1 \bmod 4, \mathscr{Q}^{+}(3, q)$ has only (up to a projectivity) the linear flock and the Thas flock. For $q=11,23,59$, other flocks of $\mathscr{Q}^{+}(3, q)$ were discovered, called exceptional flocks [1, 3, 29]. Finally, the combined results of Bader and Lunardon [2] and Thas [42] proved that very flock of $\mathscr{Q}^{+}(3, q), q$ odd, is linear, a Thas flock or one of the exceptional flocks.

The classification theorem is therefore as follows.

Theorem 8. Let $\mathscr{E}$ be a MES defined by a flock $F$ of $\mathscr{Q}^{+}(3, q)$ in the matrix model of $\operatorname{PG}(3, q)$. Then, either $q$ is even and $\mathscr{E}$ is a line, or $q$ is odd and one of the following possibilities occurs:

(1) $\mathscr{E}$ is a line;

(2) $\mathscr{E}$ consists of $(q+1) / 2$ points on two lines $\ell, \ell^{\perp}$, where $\perp$ is the polarity of $\mathscr{Q}^{+}(3, q)$;

(3) $\mathscr{E}$ is one of the sporadic examples.

In our setting, the linear MES corresponds to a $(2,2,1) \mathbb{F}_{q}$-linear MRD-code. In all the other instances (Theorem 8 (2) and (3)), the MES corresponds to a $(2,2,1)$ non-linear maximum rank distance code.

\subsection{The case $n=3$}

A very useful model of $\mathscr{S}_{2,2}$ arises from the geometry of the Desarguesian projective plane $\pi:=\operatorname{PG}\left(2, q^{3}\right)$. Indeed, each point $P$ of $\operatorname{PG}\left(2, q^{3}\right)$, when read over $\mathbb{F}_{q}$, defines a projective plane $X(P)$ of the projective space $\operatorname{PG}(8, q)$, and the set $\mathscr{D}=\left\{X(P): P \in \mathrm{PG}\left(2, q^{3}\right)\right\}$ is a Desarguesian spread of $\mathrm{PG}(8, q)$ [38, Section 25]. The incidence structure $\pi:=(\mathscr{D}, \mathscr{L})$, whose points are the elements of $\mathscr{D}$ and whose line set $\mathscr{L}$ consists of the 5-dimensional projective subspaces of $\operatorname{PG}(8, q)$ joining any two distinct elements of $\mathscr{D}$, is isomorphic to $\operatorname{PG}\left(2, q^{3}\right)$. The pair $(\mathscr{D}, \mathscr{L})$ is called the $\mathbb{F}_{q}$-linear representation of $\operatorname{PG}\left(2, q^{3}\right)$ (with respect to the Desarguesian spread $\mathscr{D}$ ).

Let $X_{0}, X_{1}, X_{2}$ denote projective homogeneous coordinates in $\pi \simeq \mathrm{PG}\left(2, q^{3}\right)$ and let $\bar{\pi}$ be a subplane of $\pi$ of order $q$. Let $G$ denote the stabilizer of $\bar{\pi}$ in $\operatorname{PGL}\left(3, q^{3}\right)$.

Choose homogeneous coordinates in such a way that $\bar{\pi}:=\left\{\left(1, x^{q+1}, x^{q}\right): x \in\right.$ $\left.\mathbb{F}_{q^{3}} \backslash\{0\}, N(x)=1\right\}$, where $N(\cdot)$ is the norm function from $\mathbb{F}_{q^{3}}$ over $\mathbb{F}_{q}$. It turns out that $\bar{\pi}$ is fixed pointwise by the order three semilinear collineation of $\operatorname{PG}\left(2, q^{3}\right)$ given by $\phi:\left(X_{0}, X_{1}, X_{2}\right) \mapsto\left(X_{2}^{q}, X_{0}^{q}, X_{1}^{q}\right)$. 
Let $\langle S\rangle$ be a Singer cyclic group of $G$ [28]. We can assume that $S$ is given by

$$
\left(\begin{array}{ccc}
\omega & 0 & 0 \\
0 & \omega^{q} & 0 \\
0 & 0 & \omega^{q^{2}}
\end{array}\right),
$$

where $\omega$ is a primitive element of $\mathbb{F}_{q^{3}}$.

Remark 4. The subgroup $\langle S\rangle$ fixes the three points $E_{1}=(1,0,0), E_{2}=(0,1,0)$ and $E_{3}=(0,0,1)$ of $\pi$, and hence the lines $E_{i} E_{j}, 1 \leq i<j \leq 3$. All the other orbits are subplanes of order $q$ of $\pi$. Note that the line $E_{i} E_{j}$ is partitioned into the two points $E_{i}$ and $E_{j}$, and into $q-1$ orbits of $\langle S\rangle$ of size $q^{2}+q+1$. The collineation $\phi$ above normalizes $\langle S\rangle$.

The points of $\bar{\pi}$ correspond to the $q^{2}+q+1$ planes filling the system of a Segre variety $\mathscr{S}_{2,2}$ of $\mathrm{PG}(8, q)$ contained in the Desarguesian spread $\mathscr{D}$. Also, the lines of $\pi$, arising from sublines of $\bar{\pi}$, yield a set of $\left(q^{3}-q\right)\left(q^{2}+q+1\right)$ points of $\pi$ that together with the points of $\bar{\pi}$ give rise to the points of the secant variety $\Omega\left(\mathscr{S}_{2,2}\right)$ of $\mathscr{S}_{2,2}[33,35]$.

Under the action of the stabilizer $G$ of $\bar{\pi}$ in $\operatorname{PGL}\left(3, q^{3}\right)$, the point set of $\pi$ is partitioned into three orbits corresponding to the points of $\bar{\pi}$, points of $\pi \backslash \bar{\pi}$ on extended sublines of $\bar{\pi}$, and the complement. Under the same group, by duality, the line set of $\pi$ is partitioned into three orbits corresponding to sublines of $\bar{\pi}$, lines meeting $\bar{\pi}$ in a point, and lines external to $\bar{\pi}$.

Proposition 2. In the linear representation of $\mathrm{PG}\left(2, q^{3}\right)$, any line of $\pi$ disjoint from $\bar{\pi}$ corresponds to a 5-dimensional projective subspace of $\mathrm{PG}(8, q)$ disjoint from $\mathscr{S}_{2,2}$.

Of course, any line of $\pi$ disjoint from $\bar{\pi}$ gives rise to an exterior set with respect to $\mathscr{S}_{2,2}$ and hence, from a coding theoretical point of view, a $(3,3,2) \mathbb{F}_{q}$-linear MRD code.

Now let $q>2$ and consider the set $\mathscr{X}$ of points of $\pi$ whose coordinates satisfy the equation $X_{0} X_{1}^{q}-X_{2}^{q+1}=0$. The set $\mathscr{X}$ has size $q^{3}+1$ and it is fixed by $\langle S\rangle$. Also, it contains $q-1$ subplanes of order $q$, one of which is $\bar{\pi}$, and the points $E_{1}$ and $E_{2}$. More precisely, the subplanes of order $q$ embedded in $\mathscr{X}$ are the subsets of points of $\pi$ given by

$$
\pi_{a}:=\left\{\left(1, x^{q+1}, x^{q}\right): x \in \mathbb{F}_{q^{3}}, N(x)=a\right\},
$$

where $a$ is a nonzero element of $\mathbb{F}_{q}$. In particular, $\pi_{1}=\bar{\pi}$. From [16, Proposition 3.1], a line of $\pi$ intersects $\mathscr{X}$ in $0,1,2$ or $q+1$ points, and the intersections of size $q+1$ are actually lines of subplanes of order $q$ of $\pi$ embedded in $\mathscr{X}$. We can assume that the Segre variety corresponding to $\bar{\pi}=\pi_{1}$ is the only Segre variety of $\mathrm{PG}(8, q)$ corresponding to rank one matrices of order three.

We recall the following definition. 
Definition 4. [4] Let $\ell_{\infty}$ be a line of $\pi$ disjoint from the subplane $\bar{\pi}$. The exterior splash of $\bar{\pi}$ on $\ell_{\infty}$ is defined to be the set of $q^{2}+q+1$ points of $\ell_{\infty}$ that belong to an extended line of $\bar{\pi}$.

The line $E_{1} E_{2}$ is disjoint from all the $q-1$ subplanes $\pi_{a}, a \in \mathbb{F}_{q} \backslash\{0\}$, of $\pi$ contained in $\mathscr{X}$. Also, for each subplane $\pi_{a}$, with $a \in \mathbb{F}_{q} \backslash\{0\}$, its exterior splash on $E_{1} E_{2}$ is the set of $q^{2}+q+1$ points of $E_{1} E_{2}$ given by

$$
Z_{a}:=\left\{(1, x, 0): x \in \mathbb{F}_{q^{3}}, N(x)=-a^{2}\right\} .
$$

Such a set is a so-called $\mathbb{F}_{q}$-linear set of pseudoregulus type. For further details on these linear sets, see $[16,34,35]$. All these subplanes and splashes are of course $\langle S\rangle$-orbits.

Now, let $T$ be the fundamental triangle $E_{1} E_{2} E_{3}$ of $\pi$. One can prove that a line of $\pi$ is either a side of $T$, or it contains a vertex of $T$, or it induces a subline of a unique subplane of order $q$ of $\pi$ invariant under $\langle S\rangle$. Consider now the set $K:=$ $\left(\mathscr{X} \backslash\left\{\pi_{1}\right\}\right) \cup Z_{1}$. It turns out that $K$ is such that every line defined by any two of its points is disjoint from $\pi_{1}$. Correspondingly, the set $K^{\prime}$ corresponding to $K$ in $\mathrm{PG}(8, q), q>2$, is an exterior set of size $\left(q^{3}+1\right)\left(q^{2}+q+1\right)$ with respect to the Segre variety $\mathscr{S}_{2,2}$ corresponding to $\pi_{1}$.

In terms of coding theory, we have the following result.

Theorem 9. There exists a $(3,3,2)$ MRD non-linear code admitting a Singer cyclic group of $\operatorname{PGL}(3, q), q>2$, as an automorphism group.

Remark 5. In [21], R. Figueroa presented a new class of non-Desarguesian projective planes of order $q^{3}, q$ a prime power with $q \neq \equiv 1 \bmod 3, q>2$. C. Hering and H.-J. Schaffer in [23] improved and simplified the construction for all prime powers $q$. From [7, Corollary 3], the set $K$ constructed above represents a line in the Figueroa plane of order $q^{3}$.

Remark 6. When $q=2$, some computer tests performed with MAGMA [8] give that all subsets of PG $(2,8)$ yielding exterior sets with respect to a Segre variety $\mathscr{S}_{2,2}$ are precisely the 24 lines disjoint from $\bar{\pi}$. When $q=2$, no non-linear MRD codes arise from our construction.

We also refer the readers to Chapter 1 of this Springer special volume, dedicated to rank metric codes, and to Chapter 3 of this special volume on the construction of cyclic subspace codes and maximum rank distance codes.

\section{References}

1. L. Bader, Some new examples of flocks of $Q^{+}(3, q)$. Geom. Dedicata 27 (1988), 213-218.

2. L. Bader and G. Lunardon, On the flocks of $\mathscr{Q}^{+}(3, q)$. Geom. Dedicata 29 (1989), 177-183.

3. R.D. Baker and G.L. Ebert, A nonlinear flock in the minkowski plane of order 11. Congr. Numer. 58 (1987), 75-81. 
4. S.G. Barwick and W.-A. Jackson, Exterior splashes and linear sets of rank 3. Discrete Math. 339 (2016), 1613-1623.

5. A. Beutelspacher, Partial spreads in finite projective spaces and partial designs. Math. Z. 145 (1975), 211-229.

6. A. Beutelspacher, On $t$-covers in finite projective spaces. J. Geom. 12 (1979), 10-16.

7. J.M.N. Brown, Some partitions in Figueroa planes. Note Mat. 29 (2009), 33-43.

8. J. Cannon and C. Playoust, An introduction to MAGMA. University of Sydney, Sydney, Australia, 1993.

9. B.N. Cooperstein, External flats to varieties in $P G\left(M_{n, n}(G F(q))\right)$. Linear Algebra Appl. 267 (1997), 175-186.

10. A. Cossidente, G. Marino, and F. Pavese, Non-linear maximum rank distance codes. Des. Codes Cryptogr. 79 (2016), 597-609.

11. A. Cossidente and F. Pavese, On subspace codes. Des. Codes Cryptogr. 78 (2016), 527-531.

12. A. Cossidente and F. Pavese, Subspace codes in $\mathrm{PG}(2 n-1, q)$. Combinatorica (to appear) (DOI:10.1007/s00493-016-3354-5).

13. A. Cossidente, F. Pavese, and L. Storme, Optimal subspace codes in PG(4,q). (In preparation).

14. P. Delsarte, Bilinear forms over a finite field, with applications to coding theory. J. Combin. Theory, Ser. A 25 (1978), 226-241.

15. P. Dembowski, Finite Geometries. Springer, Berlin, Heidelberg, New York 1968.

16. G. Donati and N. Durante, Scattered linear sets generated by collineations between pencils of lines. J. Algebraic Combin. 40 (2014), 1121-1134.

17. J. Eisfeld and L. Storme, (Partial) $t$-spreads and minimal $t$-covers in finite projective spaces. Lecture notes Universiteit Gent (2000). Available at http://cage.ugent.be/ fdc/courses/GGaGP2.php

18. S. El-Zanati, H. Jordon, G. Seelinger, P. Sissokho, and L. Spence, The maximum size of a partial 3-spread in a finite vector space over GF(2). Des. Codes Cryptogr. 54 (2010), 101107.

19. T. Etzion and N. Silberstein, Codes and Designs Related to Lifted MRD Codes. IEEE Trans. Inform. Theory $\mathbf{5 9}$ (2013), 1004-1017.

20. T. Etzion and A. Vardy, Error-correcting codes in projective space. IEEE Trans. Inform. Theory 57 (2011), 1165-1173.

21. R. Figueroa, A family of not $(V, l)$-transitive projective planes of order $q^{3}, q \not \equiv 1 \bmod 3$ and $q>2$. Math. Z. 181 (1982), 471-479.

22. E.M. Gabidulin, Theory of codes with maximum rank distance. Problems Inform. Trans. 21 (1985), 1-12.

23. C. Hering and H.-J. Schaffer, On the new projective planes of R. Figueroa. Combinatorial theory, LNM 969 (1982), 187-190.

24. J.W.P. Hirschfeld, Projective Geometries Over Finite Fields (Second Edition). Oxford: Oxford University Press 1998.

25. J.W.P. Hirschfeld, Finite Projective Spaces of Three Dimensions. Oxford: Oxford University Press 1985.

26. J.W.P. Hirschfeld and J.A. Thas, General Galois Geometries. Oxford: Oxford University Press 1991.

27. T. Honold, M. Kiermaier, and S. Kurz, Optimal binary subspace codes of length 6 , constant dimension 3 and minimum distance 4. Contemp. Math. 632 (2015), 157-176.

28. B. Huppert, Endliche Gruppen, I. Die Grundlehren der Mathematischen Wissenschaften, Band 134 Springer-Verlag, Berlin-New York 1967.

29. N.L. Johnson, Flocks of hyperbolic quadrics and translation planes admitting affine homologies. J. Geom. 34 (1989), 50-73.

30. A. Klein, K. Metsch, and L. Storme, Small maximal partial spreads in classical finite polar spaces. Adv. Geom. 10 (2010), 379-402.

31. R. Kötter and F. Kschischang, Coding for errors and erasures in random network coding. IEEE Trans. Inform. Theory 54 (2008), 3579-3591.

32. S. Kurz, Improved upper bounds for partial spreads. (arXiv:1606.08581). 
33. M. Lavrauw and G. Van de Voorde, Field reduction and linear sets in finite geometry. Contemp. Math. 632 (2015), 271-293.

34. M. Lavrauw and C. Zanella, Subgeometries and linear sets on a projective line. Finite Fields Appl. 34 (2015), 95-106.

35. G. Lunardon, G. Marino, O. Polverino, and R. Trombetti, Maximum scattered linear sets of pseudoregulus type and the Segre Variety $\mathscr{S}_{n, n}$. J. Algebraic Combin. 39 (2014), 807-831.

36. G. Lunardon, R. Trombetti, and Y. Zhou, Generalized Twisted Gabidulin Codes. (arXiv:1507.07855)

37. E. Năstase and P. Sissokho, The maximum size of a partial spread in a finite projective space. (arXiv:1605.04824)

38. B. Segre, Teoria di Galois, fibrazioni proiettive e geometrie non desarguesiane. Ann. Mat. Pura Appl. 64 (1964), 1-76.

39. J. Sheekey, A new family of linear maximum rank distance codes. Adv. Math. Commun. 10 (2016), 475-488.

40. D. Silva, F. R. Kschischang, and R. Koetter, A rank-metric approach to error control in random network coding. IEEE Trans. Inform. Theory 54 (2008), 3951-3967.

41. J.A. Thas, Flocks of non-singular ruled quadrics in $\mathrm{PG}(3, q)$. Atti Accad. Naz. Lincei Rend. Cl. Sci. Fis. Mat. Natur. 59 (1975), 83-85.

42. J.A. Thas, Flocks, maximal exterior sets and inversive planes. Finite Geometries and Combinatorial Designs, Contemporary Mathematics, Vol. 111. American Mathematical Society, Providence RI (1990), pp. 187-218. 